\title{
Optimal consumer search with prospect utility in hybrid uncertain environment
}

\author{
Chi Zhou, Wansheng Tang* and Ruiqing Zhao
}

\footnotetext{
*Correspondence: tang@tju.edu.cn Institute of Systems Engineering, Tianjin University, Tianjin 300072, China
}

\begin{abstract}
This paper studies a consumer search model with prospect utility in a hybrid uncertain environment. Hybrid uncertainty consists of the uncertainty of the consumer's valuation for each product and the randomness of stockout. An optimal search strategy is designed for the consumer to search or buy a product. The results show that the presence of prospect utility can result in low purchase threshold. By comparative statics, we demonstrate that the consumer's purchase threshold for low-value product increases with the risk coefficients for gains and losses, while the purchase threshold for high-value product decreases with the risk coefficients for gains and losses. In addition, we find that the consumer who is more sensitive to the loss will search for much less products. Finally, some numerical examples are given to illustrate the relationships between the model without and with prospect utility and further verify the effectiveness and credibility of the conclusions.
\end{abstract}

Keywords: Consumer search; Prospect theory; Loss aversion; Optimal stopping; Uncertain random variable

\section{Introduction}

Reference points and the different perceptions of gains and losses play an important role in actual consumer economics [1]. In a commodity market, consumers' purchase decisions depend not only on their valuations for the current product but also on their reference valuations for the past observed product. Kahneman and Tversky [2] and Tversky and Kahneman $[3,4]$ suggest that the perception of potential losses may be much more important in determining behavior than the perceptions of potential gains. This is known as prospect utility which is fear of loss rather than hope of gain that limits consumer's purchase behavior. These characteristics are often ignored in dynamic consumer search which do not take into account prospect utility in searching differentiated but substitutable products.

Generally speaking, there is uncertain information in the existing patterns of consumer search models. The number of differentiated but substitutable products is assumed to be finite in the market. Probability distributions of the products' value are usually assumed to be known to consumers before searching, but consumers do not know which firm will offer which product value. In the real world, some information, such as quality, pattern, and size of one product, cannot be exactly predicted by consumers in advance, thus resulting in the unknown frequencies. Therefore, probability theory is no longer applicable to

(c) 2015 Zhou et al.; licensee Springer. This is an Open Access article distributed under the terms of the Creative Commons

Attribution License (http://creativecommons.org/licenses/by/4.0), which permits unrestricted use, distribution, and reproduction in any medium, provided the original work is properly credited. 
characterize such kind of uncertain information, because the lack of the frequency leads to the unknowable probability distribution. Since the valuation of the consumer to each product is subjective and the products are heterogeneous, the valuations can be characterized as independent but not identically distributed uncertain variables [5]. Moreover, products are randomly out of stock at a point in time. This is a realistic assumption. For example, a study of more than 71,000 consumers in 29 countries shows that they have little patience for stockouts.Once facing with a stockout, $21 \%$ to $43 \%$ of consumers will actually go to another store to buy a substitute product ${ }^{\mathrm{a}}$. The valuation is an uncertain variable and the availability of previous product is random; thus, the maximum valuation between the current product and the best previous product can be characterized as an uncertain random variable [6]. Hence, the so-called twofold uncertainty is involved in this paper which consists of the uncertain product value and the random stockout.

This paper provides a simple explanation for how prospect utility affects consumers' purchase behaviors in a hybrid uncertain environment. We study a consumer search model with prospect utility in which the consumer searches sequentially and evaluates each product on the basis of multiple attributes. The multiple attributes of a product include the price, quality, color, pattern, material, and size of a product [7]. In the search process, the consumer exhibits loss aversion and decides when to stop searching for new substitute products by buying one of the products which have been encountered so far. An optimal stopping rule and an optimal search strategy are presented to maximize the benefit of the consumer. To discuss the effects of prospect utility on purchase threshold, we make a comparison between the model without and with prospect utility. By comparative statics, we further investigate the effects of risk coefficients for gains and losses, coefficient of loss aversion on the consumer's purchase threshold.

As a result of intercomparison between the model without and with prospect utility, we find that the purchase decisions of a consumer under prospect utility exhibit low purchase levels. Moreover, we show that the consumer's purchase threshold is decreasing with the search cost and is increasing with the probability of stockout. The product that has a good reputation will be preferred in the optimal search strategy. Our analysis also reveals some intriguing comparative statics. In our model, the consumer's purchase threshold for low-value product ${ }^{\mathrm{b}}$ monotonically increases as the risk coefficients for gains and losses become larger; the purchase threshold for high-value product decreases as the risk coefficients for gains and losses increase, holding other parameters of the model constant. Our findings also offer a theoretical explanation of the experimental evidence that consumers generally stop searching too early [8]. We demonstrate the consumer's purchase threshold is decreasing with the coefficient of loss aversion. This implies that if loss aversion is larger, the consumer will search for much less products because of low purchase threshold. Finally, our numerical analysis shows that the reservation value in the model with prospect utility will be lower than that in the model without prospect utility in a wider number range. These results further illustrate the feasibility of the comparative statics.

The remainder of this article is organized as follows: In the 'Literature review' section, we present our literature review. The 'Preliminaries' section recalls some fundamental concepts and formulas about uncertainty theory and chance theory. 'The model without prospect utility' section describes the consumer search model without prospect utility. 'The model without prospect utility' section studies the same model with prospect utility. We present an optimal search strategy and investigate the relationship between the 
reservation values without and with prospect utility. The 'Comparative statics' section discusses the effects of prospect utility's parameters on the reservation value. The numerical examples are provided in the 'Numerical examples' section, and we conclude in the ‘Conclusion' section.

\section{Literature review}

The consumer search problem has attracted wide attention in the academic literature. Stahl [9] discussed a simple consumer search problem in which consumers search sequentially with perfect recall and a finite number of identical stores compete by choosing prices for a homogeneous good. Cachon et al. [10] studied a model in which consumers search among multiple competing firms for products. Branco et al. [7] established a continuoustime search model of gradual learning, in which consumers incur search costs to learn product information and update their expected utility of the product at each search occasion. Arbatskaya [11] presented an ordered search model that yields an equilibrium in which there is price dispersion, prices and profits decline in the order of search, and consumers with lower search costs search longer and obtain better deals. Based on the ordered search model, Wilson [12] studied a problem that consumers are allowed to choose the optimal order in which to search firms and firms are able to obfuscate this order by deliberately increasing search costs and prices. Zhou [13] improved the ordered search model in which consumers search both for price and product fitness. Chen and Zhang [14] considered a consumer search model in a homogeneous product market where uninformed consumers search for price information. Haan and Moraga-González [15] studied the model that when consumers search for products, they first visit the firm whose advertising is most salient. Chen and He [16] studied a model of product differentiation in which the auction of advertisement positions is embedded in a market game of consumer search.

Recent research trends in consumer search investigate the issue of boundedly rational consumer behavior. For example, Hardie et al. [17] developed a multinomial logit formulation of a reference-dependent choice model. They assumed that consumer choice is influenced by the position of brands relative to multiattribute reference points and loss aversion is exhibited. Weng [18] considered the sequential search problem where sellers search for the best price from a known distribution and exhibit asymmetric regretting and rejoicing. Zhou [19] considered a consumer search model and examined the implications of consumer reference dependence for market competition. He also finds that consumer loss aversion in the price dimension intensifies competition while that in the product dimension softens competition. Kuksov and Wang [20] also examined how the competitive strategy and profitability of firms are affected by the presence of consumer loss aversion in the price dimension. The above literature has neither studied any consumer's search strategy in conjunction with nonlinear prospect utility nor discussed the probabilistic stockout of products.

The prospect theory originates in the work of Kahneman and Tversky [2]. The most prominent feature of prospect theory is loss aversion, that is, people care much more about losses relative to their reference level than about gains. It (in particular, loss aversion) has been widely applied to management and operations fields. For example, Bowman et al. [21] studied a consumption-savings model based on loss aversion that implies a fundamental asymmetry in consumption behavior. Popescu and $\mathrm{Wu}$ [22] 
considered the dynamic pricing problem of a monopolist firm in a market with repeated interactions. They presented purchase decisions by assessing prices as discounts or surcharges relative to the reference price in the spirit of prospect theory. Foellmi et al. [23] studied the Cass-Koopmans-Ramsey model of optimal economic growth in the presence of loss aversion and habit formation. They showed that prospect utility can cause the economy to remain in a steady state with low consumption and low capital.

The existing literature depicted the uncertain information in consumer search problems by random variable. However, due to the lack of historical data and the impact of subjective factor, describing the uncertainty as randomness is not adequately reasonable. Uncertain measure is an alternative tool to characterize such uncertain information that has no any historical data and cannot be exactly predicted in advance. Liu [5] gave the basic concepts about uncertainty theory, such as uncertain variable, uncertainty distribution, expected value, and variance. From then on, uncertainty theory attracts considerable attention among researchers in many fields as an important role of mathematical model to deal with human uncertainty. For instance, $\mathrm{Mu}$ [24] studied the implementation of the employment relationship problem between the enterprise and the rural migrant worker, where enterprise's assessment on the rural migrant worker's own income at home is characterized as an uncertain variable. Lan and $\mathrm{Du}$ [25] considered a warranty contracting problem faced by a buyer who purchases products from a supplier and then sells them to consumers under uncertain demand. Zhou et al. [26] studied a dynamic recruitment problem with enterprise performance in the uncertain environment and presented an optimal search strategy for the firm's employment decisions. Wang et al. [27] considered a wage contract design problem faced by an employer who employs an employee to work in labor market and characterized the employee's ability as an uncertain variable. Wu et al. [28] studied the agency problem with multiple uncertain information. They introduced confidence level to quantify the degree of the agent's risk aversion and examined the effects of the agent's attitude toward risk on the optimal contract and the principal's income.

As an important extension of uncertainty theory, chance theory was pioneered by Liu [6] for modeling complex systems with not only uncertainty but also randomness. And then, many researchers proposed uncertain random programming [29], uncertain random process [30], uncertain random graph, and uncertain random network [31]. In addition, Sheng and Yao [32] provided some formulas to calculate the variance of uncertain random variables through chance distribution and inverse chance distribution. Zhou et al. [33] proposed uncertain random multi-objective programming for optimizing multiple, non-commensurable, and conflicting objectives.

\section{Preliminaries}

This section will introduce some basic results in uncertainty theory and chance theory. The former is a branch of axiomatic mathematics for modeling belief degrees, and the latter is a methodology for modeling complex systems with not only uncertainty but also randomness.

\section{Uncertainty theory}

Let $\Gamma$ be a nonempty set and $\mathcal{L}$ a $\sigma$-algebra over $\Gamma$. Each element $\Lambda$ in $\mathcal{L}$ is called an event. Liu [5] defined an uncertain measure by the following axioms: 
Axiom 1. (Normality axiom) $\mathcal{M}\{\Gamma\}=1$ for the universal set $\Gamma$.

Axiom 2. (Duality axiom) $\mathcal{M}\{\Lambda\}+\mathcal{M}\left\{\Lambda^{c}\right\}=1, \forall \Lambda \in \mathcal{L}$.

Axiom 3. (Subadditivity axiom) $\mathcal{M}\left\{\bigcup_{i=1}^{\infty} \Lambda_{i}\right\} \leq \sum_{i=1}^{\infty} \mathcal{M}\left\{\Lambda_{i}\right\}, \forall \Lambda_{i} \in \mathcal{L}, i=1,2, \ldots$

The triplet $(\Gamma, \mathcal{L}, \mathcal{M})$ is called an uncertainty space. Furthermore, Liu [34] defined a product uncertain measure by the fourth axiom:

Axiom 4. (Product axiom) Let $\left(\Gamma_{k}, \mathcal{L}_{k}, \mathcal{M}_{k}\right)$ be uncertainty spaces for $k=1,2, \ldots$ Then, the product uncertain measure $\mathcal{M}$ is an uncertain measure satisfying:

$$
\mathcal{M}\left\{\prod_{k=1}^{\infty} \Lambda_{k}\right\}=\bigwedge_{k=1}^{\infty} \mathcal{M}_{k}\left\{\Lambda_{k}\right\}
$$

where $\Lambda_{k}$ are arbitrarily chosen events from $\mathcal{L}_{k}$ for $k=1,2, \ldots$, respectively.

An uncertain variable was defined by Liu [5] as a function $\xi$ from an uncertainty space $(\Gamma, \mathcal{L}, \mathcal{M})$ to the set of real numbers such that $\{\xi \in B\}=\{v \in \Gamma \mid \xi(v) \in B\}$ is an event for any Borel set $B$. The uncertainty distribution $\Phi$ of an uncertain variable $\xi$ was defined by Liu [5] as $\Phi(x)=\mathcal{M}\{\xi \leq x\}$ for any real number $x$. Peng and Iwamura [35] verified that a function $\Phi: \mathcal{R} \rightarrow[0,1]$ is an uncertainty distribution if and only if it is a monotone increasing function except $\Phi(x) \equiv 0$ and $\Phi(x) \equiv 1$. An uncertainty distribution $\Phi(x)$ is said to be regular if it is a continuous and strictly increasing function with respect to $x$ at which $0<\Phi(x)<1$, and:

$$
\lim _{x \rightarrow-\infty} \Phi(x)=0, \lim _{x \rightarrow+\infty} \Phi(x)=1 .
$$

Let $\xi$ be an uncertain variable with regular uncertainty distribution $\Phi(x)$. The inverse function $\Phi^{-1}(\alpha)$ is called the inverse uncertainty distribution of $\xi$ [36]. Liu [37] also verified that a function $\Phi^{-1}(\alpha):(0,1) \rightarrow \mathcal{R}$ is an inverse uncertainty distribution if and only if it is a continuous and strictly increasing function with respect to $\alpha$.

The expected value of an uncertain variable $\xi$ was defined by Liu [5] as follows:

$$
\mathrm{E}[\xi]=\int_{0}^{+\infty} \mathcal{M}\{\xi \geq r\} \mathrm{d} r-\int_{-\infty}^{0} \mathcal{M}\{\xi \leq r\} \mathrm{d} r
$$

provided that at least one of the two integrals is finite. If $\xi$ has an uncertainty distribution $\Phi$, then the expected value may be calculated by:

$$
\mathrm{E}[\xi]=\int_{0}^{+\infty}(1-\Phi(x)) \mathrm{d} x-\int_{-\infty}^{0} \Phi(x) \mathrm{d} x,
$$

or equivalently, $\mathrm{E}[\xi]=\int_{-\infty}^{+\infty} x \mathrm{~d} \Phi(x)$. If $\Phi$ is also regular, then:

$$
\mathrm{E}[\xi]=\int_{0}^{1} \Phi^{-1}(\alpha) \mathrm{d} \alpha .
$$

By the literature [34], the uncertain variables $\xi_{1}, \xi_{2}, \ldots, \xi_{n}$ are said to be independent if:

$$
\mathcal{M}\left\{\bigcap_{i=1}^{n}\left(\xi_{i} \in B_{i}\right)\right\}=\bigwedge_{i=1}^{n} \mathcal{M}\left\{\xi_{i} \in B_{i}\right\}
$$

for any Borel sets $B_{1}, B_{2}, \ldots, B_{n}$ of real numbers. Moreover, if $\xi$ and $\eta$ are independent uncertain variables with finite expected values, then:

$$
\mathrm{E}\left[d_{1} \xi+d_{2} \eta\right]=d_{1} \mathrm{E}[\xi]+d_{2} \mathrm{E}[\eta]
$$

for any real numbers $d_{1}$ and $d_{2}$, which is referred to as the linearity of expected value operator. 
Lemma 1. (Liu [36]) Let $\xi_{1}, \xi_{2}, \ldots, \xi_{n}$ be independent uncertain variables with regular uncertainty distributions $\Phi_{1}, \Phi_{2}, \ldots, \Phi_{n}$, respectively. If the function $f\left(x_{1}, x_{2}, \ldots, x_{n}\right)$ is strictly increasing with respect to $x_{1}, x_{2}, \ldots, x_{m}$ and strictly decreasing with respect to $x_{m+1}, x_{m+2}, \ldots, x_{n}$, then $\xi=f\left(\xi_{1}, \xi_{2}, \ldots, \xi_{n}\right)$ is also an uncertain variable with the inverse uncertainty distribution:

$$
\Psi^{-1}(\alpha)=f\left(\Phi_{1}^{-1}(\alpha), \ldots, \Phi_{m}^{-1}(\alpha), \Phi_{m+1}^{-1}(1-\alpha), \ldots, \Phi_{n}^{-1}(1-\alpha)\right) .
$$

Liu and $\mathrm{Ha}[38]$ proved that the uncertain variable $\xi$ has an expected value:

$$
\mathrm{E}[\xi]=\int_{0}^{1} f\left(\Phi_{1}^{-1}(\alpha), \ldots, \Phi_{m}^{-1}(\alpha), \Phi_{m+1}^{-1}(1-\alpha), \ldots, \Phi_{n}^{-1}(1-\alpha)\right) \mathrm{d} \alpha .
$$

Lemma 2. (Liu and Ha [38]) Let $\xi$ be an uncertain variable on the uncertainty space $(\Theta, \mathcal{L}, \mathcal{M})$ with uncertain distribution $\Phi(x)$. If $g: \mathcal{R} \rightarrow \mathcal{R}$ is a monotone function, the expect value of $g(\xi)$ is:

$$
\mathrm{E}[g(\xi)]=\int_{-\infty}^{+\infty} g(x) d \Phi(x) .
$$

Lemma 3. (Zhou et al. [26]) Let $\xi$ be an uncertain variable with uncertainty distribution function $\Phi(\cdot)$. Assume that the support of $\xi$ is $[a, b]$, we have:

$$
\mathrm{E}[\max \{\xi, z\}]=b-z \Phi(z)-\int_{z}^{b} \Phi(x) d x
$$

where $0 \leq a \leq z \leq b$.

The definition of first-order uncertain dominance [39] is based on the uncertainty distribution functions of uncertain variables as follows:

Definition 1. Let $\zeta_{1}$ and $\zeta_{2}$ be two uncertain variables with the uncertainty distribution functions $\Psi_{1}(x)$ and $\Psi_{2}(x)$, respectively. We say that $\zeta_{1}$ uncertainly dominates $\zeta_{2}$ in the first order, denoted by $\zeta_{1} \succeq \zeta_{2}$, if and only if $\Psi_{1}(x) \leq \Psi_{2}(x)$ for all $x \in \mathcal{R}$.

\section{Chance theory}

Let $(\Gamma, \mathcal{L}, \mathcal{M})$ be an uncertainty space and let $(\Omega, \mathcal{A}, \operatorname{Pr})$ be a probability space. Then, the product $(\Gamma, \mathcal{L}, \mathcal{M}) \times(\Omega, \mathcal{A}, \operatorname{Pr})$ is called a chance space. Essentially, it is another triplet $(\Gamma \times \Omega, \mathcal{L} \times \mathcal{A}, \mathcal{M} \times \operatorname{Pr}$ ), where $\Gamma \times \Omega$ is the universal set, $\mathcal{L} \times \mathcal{A}$ is the product $\sigma$-algebra, and $\mathcal{M} \times \operatorname{Pr}$ is the product measure.

The universal set $\Gamma \times \Omega$ is the set of all ordered pairs of the form $(v, \omega)$, where $v \in \Gamma$ and $\omega \in \Omega$. That is, $\Gamma \times \Omega=\{(\nu, \omega) \mid v \in \Gamma, \omega \in \Omega\}$. The product $\sigma$-algebra $\mathcal{L} \times \mathcal{A}$ is the smallest $\sigma$-algebra containing measurable rectangles of the form $\Lambda \times A$, where $\Lambda \in \mathcal{L}$ and $A \in \mathcal{A}$. Any element $\Theta$ in $\mathcal{L} \times \mathcal{A}$ is called an event in the chance space. Then, the chance measure of $\Theta$ was defined by Liu [6] as:

$$
\operatorname{Ch}\{\Theta\}=\int_{0}^{1} \operatorname{Pr}\{\omega \in \Omega \mid \mathcal{M}\{v \in \Gamma \mid(v, \omega) \in \Theta\} \geq x\} d x .
$$

Liu [6] proved that the chance measure $\operatorname{Ch}\{\Theta\}$ is a monotone increasing function of $\Theta$ and $\operatorname{Ch}\{\Lambda \times A\}=\mathcal{M}\{\Lambda\} \times \operatorname{Pr}\{A\}$ for any $\Lambda \in \mathcal{L}$ and any $A \in \mathcal{A}$. Especially, it holds that $\operatorname{Ch}\{\emptyset\}=0, \operatorname{Ch}\{\Gamma \times \Omega\}=1$. Liu [6] also proved that the chance measure is self-dual. That is, for any event $\Theta$, we have $\operatorname{Ch}\{\Theta\}+\operatorname{Ch}\left\{\Theta^{c}\right\}=1$. Furthermore, Hou [40] proved that the chance measure is subadditive.

An uncertain random variable was defined by Liu [6] as a function $\eta$ from a chance space $(\Gamma, \mathcal{L}, \mathcal{M}) \times(\Omega, \mathcal{A}, \operatorname{Pr})$ to the set of real numbers such that $\{\eta \in B\}$ is an event 
in $\mathcal{L} \times \mathcal{A}$ for any Borel set $B$. In addition, Liu [6] defined the chance distribution of an uncertain random variable $\eta$ as $\Phi(x)=\operatorname{Ch}\{\eta \leq x\}$ for any $x \in \mathcal{R}$.

Assume $\kappa_{1}, \kappa_{2}, \ldots, \kappa_{m}$ are independent random variables with probability distributions $K_{1}, K_{2}, \ldots, K_{m}$, and $\tau_{1}, \tau_{2}, \ldots, \tau_{n}$ are independent uncertain variables with uncertainty distributions $\Upsilon_{1}, \Upsilon_{2}, \ldots, \Upsilon_{n}$, respectively. Liu [29] proved that the uncertain random variable:

$$
\eta=f\left(\kappa_{1}, \ldots, \kappa_{m}, \tau_{1}, \ldots, \tau_{n}\right)
$$

has a chance distribution $\Phi(x)=\int_{\mathcal{R}^{m}} F\left(x ; y_{1}, \ldots, y_{m}\right) \mathrm{d} K_{1}\left(y_{1}\right) \ldots \mathrm{d} K_{m}\left(y_{m}\right)$, where $F\left(x ; y_{1}, \ldots, y_{m}\right)$ is the uncertainty distribution of uncertain variable:

$$
f\left(y_{1}, \ldots, y_{m}, \tau_{1}, \ldots, \tau_{n}\right)
$$

for any real numbers $y_{1}, \ldots, y_{m}$.

Lemma 4. (Liu [29]) Let $\kappa_{1}, \kappa_{2}, \ldots, \kappa_{m}$ be independent random variables with probability distributions $K_{1}, K_{2}, \ldots, K_{m}$, respectively, and let $\tau_{1}, \tau_{2}, \ldots, \tau_{n}$ be uncertain variables (not necessarily independent), then the uncertain random variable:

$$
\eta=f\left(\kappa_{1}, \ldots, \kappa_{m}, \tau_{1}, \ldots, \tau_{n}\right)
$$

has an expected value:

$$
\mathrm{E}[\eta]=\int_{\mathcal{R}^{m}} \mathrm{E}\left[f\left(y_{1}, \ldots, y_{m}, \tau_{1}, \ldots, \tau_{n}\right)\right] \mathrm{d} K_{1}\left(y_{1}\right) \ldots \mathrm{d} K_{m}\left(y_{m}\right),
$$

where $E\left[f\left(y_{1}, \ldots, y_{m}, \tau_{1}, \ldots, \tau_{n}\right)\right]$ is the expected value of the uncertain variable $f\left(y_{1}, \ldots, y_{m}, \tau_{1}, \ldots, \tau_{n}\right)$ for any real numbers $y_{1}, \ldots, y_{m}$.

\section{The model without prospect utility}

In this section, the consumer search model without prospect utility is presented in a hybrid uncertain environment. Furthermore, the optimal stopping rule and optimal search strategy are proposed, and we discuss how the search cost, the probability of stockout, and the risky ordering of products affect the consumer's purchase threshold.

Consider a consumer who seeks sequentially to buy the best selection among a finite number $n \geq 2$ of differentiated but substitutable products indexed by $1,2, \ldots, n$. The consumer must incur a search $\operatorname{cost} c_{i}$ in order to search product $i$ and evaluate it, $\forall i \in$ $\{1,2, \ldots, n\}$. It is assumed that the consumer searches sequentially, such that a consumer is able to evaluate product $i$ before deciding whether or not to search the next product. The subjective valuation of the consumer to product $i$ is assumed to be an uncertain variable $\xi_{i}$ with support $[a, b]$, where $0 \leq a<b<+\infty$. Denote by $\Phi_{i}(x)$ the uncertainty distribution of $\xi_{i}$ and let $\phi_{i}(x)=\mathrm{d} \Phi_{i}(x) / \mathrm{d} x$. Search is also with limited recall. Having searched product $i$, he/she is free to return to buy its product at any later point only if product $i$ is in stock with probability $p$. The consumer has two choices, one is to continue searching, i.e., he/she would compare the current product with the best previous product and choose a better one as a reserved product; alternatively, he/she can stop searching and buy the reserved product.

Let the collection $I=\{1,2, \ldots, n\}$ be partitioned into the set $Q$ of encountered products and its complement $\bar{Q}$ of not encountered products. That is, $Q \cup \bar{Q}=I$, and $Q \cap \bar{Q}=\varnothing$. 
Let $z$ be the valuation of the reserved product when the set of encountered products is Q, i.e.,

$$
z=\max _{i \in Q}\left\{\hat{\xi}_{i}\right\}
$$

where $\hat{\xi}_{i}$ is a realization of $\xi_{i}$. For ease of exposition, the product with valuation $z$ is simply expressed as the reserved product $z$. Let $V(z, Q)$ represent the value function of the consumer, with the choice variable being a binary decision between stopping or continuing the search when the set of encountered products is $Q$. Define $\Pi(z, Q)$ as the consumer's benefit from choosing to stop now. When the consumer buys a product, we suppose that the benefit is equal to the subjective valuation of the product. That is to say,

$$
\Pi(z, Q)=z \text {. }
$$

The objective of the consumer is to find an optimal search strategy maximizing the benefit from the search. Such a problem can be formally described in a dynamic programming format [41]. Therefore, we can express the value function $V(z, Q)$ as follows:

$$
V(z, Q)=\max \left\{\Pi(z, Q), \max _{i \in \bar{Q}}\left\{\beta E\left[V\left(\eta_{i}, Q \cup\{i\}\right)\right]-c_{i}\right\}\right\},
$$

where $\eta_{i}=\max \left\{\xi_{i}, z\right\}$ is an uncertain random variable and $\beta$ denotes a discount factor with $0<\beta<1$. That is, the reserved product $z$ may be unpurchasable due to out of stock with probability $1-p$, and the reserved product $z$ may be purchasable with probability $p$.

According to Zhou et al. [26] and Lippman and McCall [42], the solution to the above multi-period dynamic programming problem is equivalent to solving a one-period static problem satisfying myopic property.

Starting from a point in time, we suppress the time dimension of the problem. To a consumer, the benefit from stopping the search is $\Pi_{\text {stop }}(z)=z$. If the consumer continues the search and the realized valuation of the next product is $\max \left\{\hat{\xi}_{i}, z\right\}$, the ex post benefit from continuing the search is:

$$
\Pi_{\text {search }}\left(\hat{\xi}_{i}, z\right)=\beta \max \left\{\hat{\xi}_{i}, z\right\}-c_{i} .
$$

Therefore, the expected benefit from continuing the search becomes:

$$
\begin{aligned}
\Pi_{\text {search }}(z) & =\mathrm{E}\left[\Pi_{\text {search }}\left(\xi_{i}, z\right)\right]=\beta \mathrm{E}\left[\max \left\{\xi_{i}, z\right\}\right]-c_{i} \\
& =\beta p \mathrm{E}\left[\max \left\{\xi_{i}, z\right\}\right]+\beta(1-p) \mathrm{E}\left[\max \left\{\xi_{i}, 0\right\}\right]-c_{i} .
\end{aligned}
$$

By Lemma 3, Equation 5 can be rewritten as follows:

$$
\Pi_{\text {search }}(z)=\beta b-\beta p z \Phi_{i}(z)-\beta \int_{z}^{b} \Phi_{i}(x) \mathrm{d} x-\beta(1-p) \int_{a}^{z} \Phi_{i}(x) \mathrm{d} x-c_{i} .
$$

By the myopic property of the optimal search strategy, we define a difference function as follows:

$$
\begin{aligned}
D(z) & \triangleq \Pi_{\text {search }}(z)-\Pi_{\text {stop }}(z) \\
& =\beta b-z-\beta p z \Phi_{i}(z)-\beta \int_{z}^{b} \Phi_{i}(x) \mathrm{d} x-\beta(1-p) \int_{a}^{z} \Phi_{i}(x) \mathrm{d} x-c_{i} .
\end{aligned}
$$

Note that:

$$
\frac{\mathrm{d} D(z)}{\mathrm{d} z}=-1-\beta p z \phi_{i}(z)<0 .
$$


Assume that $D(a)>0$ and $D(b)<0$. Since $D(z)$ is decreasing with $z$, the solution $z_{i}^{L N}$ of the equation $D\left(z_{i}^{L N}\right)=0$ exists uniquely. Thus, the critical number $z_{i}^{L N}$ which satisfies:

$$
\beta b-z_{i}^{L N}-\beta p z_{i}^{L N} \Phi_{i}\left(z_{i}^{L N}\right)-\beta \int_{z_{i}^{L N}}^{b} \Phi_{i}(x) \mathrm{d} x-\beta(1-p) \int_{a}^{z_{i}^{L N}} \Phi_{i}(x) \mathrm{d} x-c_{i}=0
$$

is called the reservation value of product $i$.

Remark. If the consumer has historical data of the product value, then the valuation may be rationally characterized as random variable. A model similar to Equation 3 can be built to maximize the consumer's expected benefit. Suppose that the probability distribution function of the product $i$ 's valuation is $F_{i}(x)$. The other parameters and assumptions are similar to those of the model without prospect utility. However, the reservation value $z_{i}^{*}$ satisfies:

$$
\beta b-z_{i}^{*}-\beta \int_{a}^{b} F_{i}(x) \mathrm{d} x+\beta p \int_{a}^{z_{i}^{*}} F_{i}(x) \mathrm{d} x-c_{i}=0 .
$$

According to the properties of reservation value, the optimal stopping rule and the optimal search strategy can be prescribed as follows:

Theorem 1. (Optimal stopping rule) If $D(z) \leq 0$, stop the search by buying the better product between the current product and the reserved product $z$, or else continue the search.

The optimal search strategy specifies the order in which alternative reserved products should be evaluated in detail and at which point evaluations should stop and a product be purchased. This strategy is specified and proven to be optimal in Weitzman [43] and involves the following procedure:

Step 1. Rank the products according to their respective reservation value.

Step 2. Evaluate the products in this order.

Step 3. Buy the first product which obtained score after the detailed evaluation is greater than the reservation value of the next product to be evaluated.

To discuss more properties of the reservation value, we obtain the following proposition:

\section{Proposition 1.}

1) The reservation value is decreasing with respect to the search cost.

2) The reservation value is increasing with respect to the probability of unpurchasable.

3) If product $\xi_{i}$ uncertainly dominates product $\xi_{j}$ in the first order and $c_{i}=c_{j}$, $\forall i, j \in I$, then the reservation value $z_{i}^{L N}$ of firm $i$ 's product is larger than the reservation value $z_{j}^{L N}$ of product $j$.

Proof.

1) For convenience, define:

$$
H_{i}(z)=\beta b-z-\beta p z \Phi_{i}(z)-\beta \int_{z}^{b} \Phi_{i}(x) \mathrm{d} x-\beta(1-p) \int_{a}^{z} \Phi_{i}(x) \mathrm{d} x .
$$


By taking the derivative of two sides of the equation $H_{i}\left(z_{i}^{L N}\right)=c_{i}$ with respect to $c_{i}$, we have $-\frac{\partial z_{i}^{L N}}{\partial c_{i}}-\beta p z_{i}^{L N} \phi_{i}\left(z_{i}^{L N}\right) \frac{\partial z_{i}^{L N}}{\partial c_{i}}=1$. Furthermore, $\frac{\partial z_{i}^{L N}}{\partial c_{i}}=$ $\frac{1}{-1-\beta p z_{i}^{L N} \phi_{i}\left(z_{i}^{L N}\right)}<0$. Therefore, the reservation value $z_{i}^{L N}$ is decreasing with the search cost $c_{i}$.

2) By taking the partial derivative of the variable $z_{i}^{L N}$ of equation $D\left(z_{i}^{L N}\right)=0$ with respect to $p$, we have:

$$
-\frac{\partial z_{i}^{L N}}{\partial p}-\beta z_{i}^{L N} \Phi_{i}\left(z_{i}^{L N}\right)-\beta p z_{i}^{L N} \phi_{i}\left(z_{i}^{L N}\right) \frac{\partial z_{i}^{L N}}{\partial p}+\beta \int_{a}^{z_{i}^{L N}} \Phi_{i}(x) \mathrm{d} x=0 .
$$

Furthermore,

$$
\left[1+\beta p z_{i}^{L N} \phi_{i}\left(z_{i}^{L N}\right)\right] \frac{\partial z_{i}^{L N}}{\partial p}=-\beta z_{i}^{L N} \Phi_{i}\left(z_{i}^{L N}\right)+\beta \int_{a}^{z_{i}^{L N}} \Phi_{i}(x) \mathrm{d} x .
$$

By using integration by parts,

$$
\left[1+\beta p z_{i}^{L N} \phi_{i}\left(z_{i}^{L N}\right)\right] \frac{\partial z_{i}^{L N}}{\partial p}=-\beta \int_{a}^{z_{i}^{L N}} x \phi_{i}(x) \mathrm{d} x .
$$

Therefore,

$$
\frac{\partial z_{i}^{L N}}{\partial p}=\frac{-\beta \int_{a}^{z_{i}^{L N}} x \phi_{i}(x) \mathrm{d} x}{1+\beta p z_{i}^{L N} \phi_{i}\left(z_{i}^{L N}\right)}<0
$$

Hence, the reservation value $z_{i}^{L N}$ is increasing with the probability $1-p$ of unpurchasable.

3) Since $\xi_{i} \succeq \xi_{j}$, it follows from Definition 1 that $\Phi_{i}(x) \leq \Phi_{j}(x), \forall i, j \in I$. Therefore, we can obtain $\int_{a}^{z} \Phi_{i}(x) \mathrm{d} x \leq \int_{a}^{z} \Phi_{j}(x) \mathrm{d} x$ and $\int_{z}^{b} \Phi_{i}(x) \mathrm{d} x \leq \int_{z}^{b} \Phi_{j}(x) \mathrm{d} x$. From Equation 10,

$$
\begin{aligned}
H_{i}(z) & =\beta b-z-\beta p z \Phi_{i}(z)-\beta \int_{z}^{b} \Phi_{i}(x) \mathrm{d} x-\beta(1-p) \int_{a}^{z} \Phi_{i}(x) \mathrm{d} x \\
& \geq \beta b-z-\beta p z \Phi_{j}(z)-\beta \int_{z}^{b} \Phi_{j}(x) \mathrm{d} x-\beta(1-p) \int_{a}^{z} \Phi_{j}(x) \mathrm{d} x=H_{j}(z) .
\end{aligned}
$$

Suppose that $c_{i}=c_{j}$. By Equation 11, $H_{i}\left(z_{i}^{L N}\right)=c_{i}=c_{j}=H_{j}\left(z_{j}^{L N}\right) \geq H_{j}\left(z_{i}^{L N}\right)$. Since $H_{i}(z)$ is strictly decreasing in $z$, we have $z_{i}^{L N} \geq z_{j}^{L N}$.

The result 1) in Proposition 1 shows that an increase in the search cost decreases the reservation value. That is, if the search cost is very costly, the consumer's purchase threshold will be lowered. There is widespread evidence that the search cost often influences the consumer's purchase threshold negatively. Furthermore, we demonstrate that an increase in the probability of stockout will yield higher reservation value. It implies that greater probability of stockout leads to higher purchase threshold. Finally, the result implies that the product that has a good reputation will be preferred in the search process.

\section{The model with prospect utility}

In this section, the optimal consumer search model is investigated when consumers are loss averse for a prospect utility function. As discussed before, the optimal stopping 
rule and optimal search strategy are also proposed. In addition, we contrast the two reservation values in the models with and without prospect utility.

Prospect theory has attracted a good deal of attention as an alternative to the wellestablished expected utility theory. The basic idea of prospect theory is that alternatives are evaluated by a prospect utility function in terms of gains and losses with respect to a reference point. According to the descriptive foundations of value function proposed by Kahneman and Tversky [2,4], define the prospect utility function as follows:

$$
U(h, k)=u(h)+R(u(h)-u(k)),
$$

where $u(\cdot)$ represents the instantaneous utility function, and $R(\cdot)$ represents the gain-loss utility function that satisfies the following form:

$$
R(u(h)-u(k))= \begin{cases}(u(h)-u(k))^{g}, & \text { if } u(h)-u(k) \geq 0 \\ -\gamma(u(k)-u(h))^{l}, & \text { if } u(h)-u(k)<0,\end{cases}
$$

where the parameters $0<g<1$ and $0<l<1$ represent the coefficients of risk attitude for gains and losses, respectively. As $g$ and $l$ become higher, the consumer tends to take more risks. In addition, the parameter $\gamma$ represents the coefficient of loss aversion. When $\gamma>1$, the consumer is more sensitive to the loss. It means that people have a relative distaste for losses when comparing very small losses to very small gains. There is strong empirical evidence on loss aversion, in which the loss aversion index $\gamma$ is estimated between 1.75 and 2.5 [44]. Therefore, the gain-loss utility function $R(\Delta u)$ is an S-shaped function of the reference utility gap $\Delta u=u(h)-u(k)$, with a concave kink at $\Delta u=0$.

Following the above prospect utility function, we define the prospect utility function of optimal consumer search as follows:

$$
U_{\text {search }}\left(\hat{\xi}_{i}, z\right)=\Pi_{\text {search }}\left(\hat{\xi}_{i}, z\right)+R\left(\Pi_{\text {search }}\left(\hat{\xi}_{i}, z\right)-\Pi_{\text {stop }}(z)\right),
$$

where

$$
\begin{aligned}
& R\left(\Pi_{\text {search }}\left(\hat{\xi}_{i}, z\right)-\Pi_{\text {stop }}(z)\right) \\
& = \begin{cases}\left(\Pi_{\text {search }}\left(\hat{\xi}_{i}, z\right)-\Pi_{\text {stop }}(z)\right)^{g}, & \text { if } \Pi_{\text {search }}\left(\hat{\xi}_{i}, z\right)-\Pi_{\text {stop }}(z) \geq 0 \\
-\gamma\left(\Pi_{\text {stop }}(z)-\Pi_{\text {search }}\left(\hat{\xi}_{i}, z\right)\right)^{l}, & \text { if } \Pi_{\text {search }}\left(\hat{\xi}_{i}, z\right)-\Pi_{\text {stop }}(z)<0 .\end{cases}
\end{aligned}
$$

Before the consumer decides to make the investment to search, he/she is assumed to be able to anticipate his/her feelings of gains and losses based on the realized prospect utility. When the reserved product $z$ is given, the expected utility from continuing the search can be written as follows:

$$
\begin{aligned}
U_{\text {search }}(z) & =\mathrm{E}\left[U_{\text {search }}\left(\xi_{i}, z\right)\right] \\
& =\mathrm{E}\left[\Pi_{\text {search }}\left(\xi_{i}, z\right)\right]+\mathrm{E}\left[R\left(\Pi_{\text {search }}\left(\xi_{i}, z\right)-\Pi_{\text {stop }}(z)\right)\right] \\
& =\Pi_{\text {search }}(z)+\mathrm{E}\left[R\left(\Pi_{\text {search }}\left(\xi_{i}, z\right)-\Pi_{\text {stop }}(z)\right)\right] .
\end{aligned}
$$

Since $R\left(\Pi_{\text {search }}\left(\xi_{i}, z\right)-\Pi_{\text {stop }}(z)\right)$ is an uncertain random variable, it follows from Lemma 4 that:

$$
\begin{aligned}
T_{R}(z)= & \mathrm{E}\left[R\left(\Pi_{\text {search }}\left(\xi_{i}, z\right)-\Pi_{\text {stop }}(z)\right)\right] \\
= & p \mathrm{E}\left[R\left(\beta \max \left\{\xi_{i}, z\right\}-c_{i}-z\right)\right]+(1-p) \mathrm{E}\left[R\left(\beta \xi_{i}-c_{i}-\xi_{i}\right)\right] \\
= & p \int_{\frac{z+c_{i}}{\beta}}^{b}\left(\beta x-c_{i}-z\right)^{g} \mathrm{~d} \Phi_{i}(x)-p \gamma \int_{z}^{\frac{z+c_{i}}{\beta}}\left(z-\beta x+c_{i}\right)^{l} \mathrm{~d} \Phi_{i}(x) \\
& -p \gamma \int_{a}^{z}\left(z-\beta z+c_{i}\right)^{l} \mathrm{~d} \Phi_{i}(x)-(1-p) \gamma \int_{a}^{b}\left(x-\beta x+c_{i}\right)^{l} \mathrm{~d} \Phi_{i}(x) .
\end{aligned}
$$


The first term on the right hand side of Equation 17 represents the expected gain utility from reserving the current product and continuing the search. The second term $-p \gamma \int_{z}^{\frac{z+c_{i}}{\beta}}\left(z-\beta x+c_{i}\right)^{l} \mathrm{~d} \Phi_{i}(x)$ represents the expected loss utility from buying the current product and stopping the search. The third term $-p \gamma \int_{a}^{z}\left(z-\beta z+c_{i}\right)^{l} \mathrm{~d} \Phi_{i}(x)$ represents the expected loss utility from buying the reserved product $z$ and stopping the search if it is available. The last term $-(1-p) \gamma \int_{a}^{b}\left(x-\beta x+c_{i}\right)^{l} \mathrm{~d} \Phi_{i}(x)$ is the expected loss utility from buying the current product and stopping the search if it is not available.

In a similar way without regard to the prospect utility, we consider that the expected utility from stopping the search is equivalent to the realized evaluation value $z$ of the reserved product. Hence, we have:

$$
U_{\text {stop }}(z)=\Pi_{\text {stop }}(z)=z .
$$

Similarly, we also define a difference function as follows:

$$
\begin{aligned}
D^{p}(z) & \triangleq U_{\text {search }}(z)-U_{\text {stop }}(z) \\
& =\Pi_{\text {search }}(z)+T_{R}(z)-\Pi_{\text {stop }}(z) \\
& =D(z)+T_{R}(z) .
\end{aligned}
$$

Therefore, the optimal stopping rule with prospect utility can be prescribed as follows:

Theorem 2. (Optimal stopping rule) If $D^{p}(z) \leq 0$, stop the search by buying the better product between the current product and the reserved product $z$, or else continue the search.

To derive the reservation value of optimal consumer search with prospect utility, it is easy to prove that:

$$
\frac{\mathrm{d} D^{p}(z)}{\mathrm{d} z}<0 .
$$

Note that $\frac{\mathrm{d} D(z)}{\mathrm{d} z}<0$,

$$
\begin{aligned}
& \frac{\partial\left(p \int_{\frac{z+c_{i}}{\beta}}^{b}\left(\beta x-c_{i}-z\right)^{g} \mathrm{~d} \Phi_{i}(x)\right)}{\partial z}=-p g \int_{\frac{z+c_{i}}{\beta}}^{b} \frac{1}{\left(\beta x-c_{i}-z\right)^{1-g}} \mathrm{~d} \Phi_{i}(x)<0, \\
& \frac{\partial\left(-p \gamma \int_{z}^{\frac{z+c_{i}}{\beta}}\left(z-\beta x+c_{i}\right)^{l} \mathrm{~d} \Phi_{i}(x)\right)}{{ }^{\frac{z+c_{i}}{\partial} z} \frac{1}{\left(z-\beta x+c_{i}\right)^{1-l}} \mathrm{~d} \Phi_{i}(x)+p \gamma\left(z-\beta z+c_{i}\right)^{l} \phi_{i}(z),} \\
& \frac{\partial\left(-p \gamma \int_{a}^{z}\left(z-\beta z+c_{i}\right)^{l} \mathrm{~d} \Phi_{i}(x)\right)}{\partial z} \\
& =-p \gamma l(1-\beta) \int_{a}^{z} \frac{1}{\left(z-\beta z+c_{i}\right)^{1-l}} \mathrm{~d} \Phi_{i}(x)-p \gamma\left(z-\beta z+c_{i}\right)^{l} \phi_{i}(z) .
\end{aligned}
$$

Futhermore, we obtain:

$$
\frac{\partial\left(-p \gamma \int_{z}^{\frac{z+c_{i}}{\beta}}\left(z-\beta x+c_{i}\right)^{l} \mathrm{~d} \Phi_{i}(x)\right)}{\partial z}+\frac{\partial\left(-p \gamma \int_{a}^{z}\left(z-\beta z+c_{i}\right)^{l} \mathrm{~d} \Phi_{i}(x)\right)}{\partial z}<0 .
$$

Hence, it follows from Equations 8, 21, and 22 that $\frac{\mathrm{d} D^{p}(z)}{\mathrm{d} z}<0$. It implies that an increase in the evaluation value $z$ of reserved product will decrease the expected prospect utility. Furthermore, since $D^{p}(z)$ is decreasing with $z$, the solution $z_{i}^{L A}$ of the equation $D^{p}\left(z_{i}^{L A}\right)=$ 0 also uniquely exists. Thus, the critical number $z_{i}^{L A}$ which satisfies $D\left(z_{i}^{L A}\right)+T_{R}\left(z_{i}^{L A}\right)=0$ is also the reservation value of firm $i$ 's product under the prospect utility. 
Proposition 2. If $\beta b-c_{i}<z_{i}^{L N}<b$, the reservation value in the model with prospect utility will be lower than that in the model without prospect utility.

Proof. Note that $D\left(z_{i}^{L N}\right)=0$. By Equation 19, we have:

$$
\begin{aligned}
D^{p}\left(z_{i}^{L N}\right) & =D\left(z_{i}^{L N}\right)+T_{R}\left(z_{i}^{L N}\right) \\
& =T_{R}\left(z_{i}^{L N}\right) \\
& =p \int_{a}^{b} R\left(\beta \max \left\{x, z_{i}^{L N}\right\}-c_{i}-z_{i}^{L N}\right) \mathrm{d} \Phi_{i}(x)+(1-p) \int_{a}^{b} R\left(\beta x-c_{i}-x\right) \mathrm{d} \Phi_{i}(x) \\
& =p \int_{a}^{b} R\left(\beta \max \left\{x, z_{i}^{L N}\right\}-c_{i}-z_{i}^{L N}\right) \mathrm{d} \Phi_{i}(x)-(1-p) \gamma \int_{a}^{b}\left(x-\beta x+c_{i}\right)^{l} \mathrm{~d} \Phi_{i}(x) .
\end{aligned}
$$

By using the first mean value theorem of integral, we obtain that if there exists $\bar{x} \in[a, b]$, the above equation can be rewritten as follows:

$$
D^{p}\left(z_{i}^{L N}\right)=p R\left(\beta \max \left\{\bar{x}, z_{i}^{L N}\right\}-c_{i}-z_{i}^{L N}\right) \phi_{i}(\bar{x})(b-a)-(1-p) \gamma \int_{a}^{b}\left(x-\beta x+c_{i}\right)^{l} \mathrm{~d} \Phi_{i}(x) .
$$

If $\beta b-c_{i}<z_{i}^{L N}<b$, we have $\bar{x} \leq b<\frac{z_{i}^{L N}+c_{i}}{\beta}$. To prove the assertion, we consider the following two cases:

Case 1. When $z_{i}^{L N} \leq \bar{x}<\frac{z_{i}^{L N}+c_{i}}{\beta}$, we have:

$$
\begin{aligned}
D^{p}\left(z_{i}^{L N}\right) & =p R\left(\beta \bar{x}-c_{i}-z_{i}^{L N}\right) \phi_{i}(\bar{x})(b-a)-(1-p) \gamma \int_{a}^{b}\left(x-\beta x+c_{i}\right)^{l} \mathrm{~d} \Phi_{i}(x) \\
& <-(1-p) \gamma \int_{a}^{b}\left(x-\beta x+c_{i}\right)^{l} \mathrm{~d} \Phi_{i}(x)<0 .
\end{aligned}
$$

Case 2. When $\bar{x}<z_{i}^{L N}$, we also have:

$$
\begin{aligned}
D^{p}\left(z_{i}^{L N}\right) & =p R\left(\beta z_{i}^{L N}-c_{i}-z_{i}^{L N}\right) \phi_{i}(\bar{x})(b-a)-(1-p) \gamma \int_{a}^{b}\left(x-\beta x+c_{i}\right)^{l} \mathrm{~d} \Phi_{i}(x) \\
& <-(1-p) \gamma \int_{a}^{b}\left(x-\beta x+c_{i}\right)^{l} \mathrm{~d} \Phi_{i}(x)<0 .
\end{aligned}
$$

Hence, if $\beta b-c_{i}<z_{i}^{L N}<b$, we have $D^{p}\left(z_{i}^{L N}\right)<0=D^{p}\left(z_{i}^{L A}\right)$. Since $D^{p}(z)$ is decreasing with $z$, we have $z_{i}^{L A}<z_{i}^{L N}$. The theorem is thus proven.

Proposition 2 indicates that the prospect utility leads to low reservation value. If the consumer is more loss averse, we conclude that the purchase threshold will be lower. The optimal search strategy and Proposition 1 also hold for the prospect utility case.

\section{Comparative statics}

In this section, we derive some comparative statics of how the reservation value under the prospect utility would respond to the changes in the parameters $g, l$, and $\gamma$. We first examine the effect of risk coefficient $g$ for gains on reservation value $z_{i}^{L A}$.

Proposition 3. Holding other parameters of the model constant, the reservation value of the firm's product under the prospect utility, which satisfies $a \leq z_{i}^{L A}<\beta b-c_{i}-2$, is increasing with respect to the coefficient of risk attitude for gains. And that which satisfies $\beta b-c_{i}-2 \leq z_{i}^{L A} \leq b$ is decreasing with respect to the coefficient of risk attitude for gains. 
Proof. From the previous discussion of $D^{p}(z)$, we can obtain that the function $D^{p}(z)$ is continuous, differentiable in $z$. By taking the partial derivative of the variable $z_{i}^{L A}$ of equation $D^{p}\left(z_{i}^{L A}\right)=0$ with respect to $g$, we have:

$$
\begin{aligned}
& -\frac{\partial z_{i}^{L A}}{\partial g}-\beta p z \phi_{i}(z) \frac{\partial z_{i}^{L A}}{\partial g}+p \int_{\frac{z+c_{i}}{\beta}}^{b}\left(\beta x-c_{i}-z\right)^{g} \ln \left(\beta x-c_{i}-z\right) \phi_{i}(x) \mathrm{d} x \\
& -p g \int_{\frac{z+c_{i}}{\beta}}^{b}\left(\beta x-c_{i}-z\right)^{g-1} \frac{\partial z_{i}^{L A}}{\partial g} \phi_{i}(x) \mathrm{d} x-p \gamma l \int_{z}^{\frac{z+c_{i}}{\beta}}\left(z-\beta x+c_{i}\right)^{l-1} \phi_{i}(x) \frac{\partial z_{i}^{L A}}{\partial g} \mathrm{~d} x \\
& -p \gamma l(1-\beta) \int_{a}^{z}\left(z-\beta z+c_{i}\right)^{l-1} \phi_{i}(x) \frac{\partial z_{i}^{L A}}{\partial g} \mathrm{~d} x=0
\end{aligned}
$$

by using the equation:

$$
\frac{\partial\left(\beta x-c_{i}-z\right)^{g}}{\partial g}=\left(\beta x-c_{i}-z\right)^{g} \ln \left(\beta x-c_{i}-z\right)-g\left(\beta x-c_{i}-z\right)^{g-1} \frac{\partial z}{\partial g} .
$$

Furthermore,

$$
\frac{\partial z_{i}^{L A}}{\partial g}=\frac{p \int_{\frac{z+c_{i}}{\beta}}^{b}\left(\beta x-c_{i}-z\right)^{g} \ln \left(\beta x-c_{i}-z\right) \phi_{i}(x) \mathrm{d} x}{N},
$$

where:

$$
\begin{aligned}
N= & 1+\beta p z \phi_{i}(z)+p g \int_{\frac{z+c_{i}}{\beta}}^{b}\left(\beta x-c_{i}-z\right)^{g-1} \phi_{i}(x) \mathrm{d} x \\
& +p \gamma l \int_{z}^{\frac{z+c_{i}}{\beta}}\left(z-\beta x+c_{i}\right)^{l-1} \phi_{i}(x) \mathrm{d} x+p \gamma l(1-\beta) \int_{a}^{z}\left(z-\beta z+c_{i}\right)^{l-1} \phi_{i}(x) \mathrm{d} x .
\end{aligned}
$$

Since $\ln \left(\beta x-c_{i}-z\right)$ could be positive or negative, it holds that $\frac{\partial z_{i}^{L A}}{\partial g}>0$ if $a \leq z_{i}^{L A}<$ $\beta b-c_{i}-2$, and $\frac{\partial z_{i}^{L A}}{\partial g}<0$ if $\beta b-c_{i}-2 \leq z_{i}^{L A} \leq b$.

Proposition 3 indicates that as the risk coefficient $g$ for gains becomes higher, the consumer raises his/her reservation value of low-value product and reduces his/her reservation value of high-value product. This implies that the consumer's purchase threshold for low-value product will be increased with the coefficient of risk attitude for gains, and the consumer's purchase threshold for high-value product will be decreased with the coefficient of risk attitude for gains.

Next, we examine the effect of risk coefficient $l$ for losses on reservation value $z_{i}^{L A}$.

Proposition 4. Holding other parameters of the model constant, if $a \leq z_{i}^{L A}<\frac{1-c_{i}}{1-\beta}$ and $\frac{a+b}{2} \leq \frac{1-c_{i}}{1-\beta}$, the reservation value under the prospect utility is increasing with respect to the coefficient of risk attitude for losses. If $\frac{2-c_{i}}{1-\beta}<z_{i}^{L A} \leq b$ and $\frac{1-c_{i}}{1-\beta}<\frac{a+b}{2}$, the reservation value under the prospect utility is decreasing with respect to the coefficient of risk attitude for losses.

Proof. By taking the partial derivative of the variable $z_{i}^{L A}$ of equation $D^{p}\left(z_{i}^{L A}\right)=0$ with respect to $l$, we have:

$$
\begin{aligned}
& -\frac{\partial z_{i}^{L A}}{\partial l}-\beta p z \phi_{i}(z) \frac{\partial z_{i}^{L A}}{\partial l}-p g \int_{\frac{z+c_{i}}{\beta}}^{b}\left(\beta x-c_{i}-z\right)^{g-1} \phi_{i}(x) \frac{\partial z_{i}^{L A}}{\partial l} \mathrm{~d} x \\
& -p \gamma \int_{z}^{\frac{z+c_{i}}{\beta}}\left(z-\beta x+c_{i}\right)^{l} \ln \left(z-\beta x+c_{i}\right) \phi_{i}(x) \mathrm{d} x-p \gamma l \int_{z}^{\frac{z+c_{i}}{\beta}}\left(z-\beta x+c_{i}\right)^{l-1} \phi_{i}(x) \frac{\partial z_{i}^{L A}}{\partial l} \mathrm{~d} x \\
& -p \gamma \int_{a}^{z}\left(z-\beta z+c_{i}\right)^{l} \ln \left(z-\beta z+c_{i}\right) \phi_{i}(x) \mathrm{d} x-p \gamma l(1-\beta) \int_{a}^{z}\left(z-\beta z+c_{i}\right)^{l-1} \phi_{i}(x) \frac{\partial z_{i}^{L A}}{\partial l} \mathrm{~d} x \\
& -(1-p) \gamma \int_{a}^{b}\left(x-\beta x+c_{i}\right)^{l} \ln \left(x-\beta x+c_{i}\right) \phi_{i}(x) \mathrm{d} x=0 .
\end{aligned}
$$


Furthermore,

$$
\frac{\partial z_{i}^{L A}}{\partial l}=\frac{-p \gamma \int_{z}^{\frac{z+c_{i}}{\beta}} S(x) \phi_{i}(x) \mathrm{d} x-p \gamma \int_{a}^{z} S(z) \phi_{i}(x) \mathrm{d} x-(1-p) \gamma \int_{a}^{b} S^{*}(x) \phi_{i}(x) \mathrm{d} x}{N},
$$

where:

$$
\begin{aligned}
N= & 1+\beta p z \phi_{i}(z)+p g \int_{\frac{z+c_{i}}{\beta}}^{b}\left(\beta x-c_{i}-z\right)^{g-1} \phi_{i}(x) \mathrm{d} x \\
& +p \gamma l \int_{z}^{\frac{z+c_{i}}{\beta}}\left(z-\beta x+c_{i}\right)^{l-1} \phi_{i}(x) \mathrm{d} x+p \gamma l(1-\beta) \int_{a}^{z}\left(z-\beta z+c_{i}\right)^{l-1} \phi_{i}(x) \mathrm{d} x,
\end{aligned}
$$

$S(x)=\left(z-\beta x+c_{i}\right)^{l} \ln \left(z-\beta x+c_{i}\right)$ and $S^{*}(x)=\left(x-\beta x+c_{i}\right)^{l} \ln \left(x-\beta x+c_{i}\right)$. Since $a \leq z_{i}^{L A}<\frac{1-c_{i}}{1-\beta}<\frac{2-c_{i}}{1-\beta}$, it holds that $-p \gamma \int_{z}^{\frac{z+c_{i}}{\beta}} S(x) \phi_{i}(x) \mathrm{d} x>0$. Furthermore, it follows from $z_{i}^{L A}<\frac{1-c_{i}}{1-\beta}$ that $-p \gamma \int_{a}^{z} S(z) \phi_{i}(x) \mathrm{d} x>0$. When $\frac{a+b}{2} \leq \frac{1-c_{i}}{1-\beta}$, we have $-(1-p) \gamma \int_{a}^{b} S^{*}(x) \phi_{i}(x) \mathrm{d} x>0$. Hence, if $a \leq z_{i}^{L A}<\frac{1-c_{i}}{1-\beta}$ and $\frac{a+b}{2} \leq \frac{1-c_{i}}{1-\beta}, \frac{\partial z_{i}^{L A}}{\partial l}>0$.

If $\frac{2-c_{i}}{1-\beta}<z_{i}^{L A} \leq b$, it holds that $-p \gamma \int_{z}^{\frac{z+c_{i}}{\beta}} S(x) \phi_{i}(x) \mathrm{d} x<0$. Moreover, it follows from $\frac{1-c_{i}}{1-\beta}<\frac{2-c_{i}}{1-\beta}<z_{i}^{L A}$ that $-p \gamma \int_{a}^{z} S(z) \phi_{i}(x) \mathrm{d} x<0$. When $\frac{1-c_{i}}{1-\beta}<\frac{a+b}{2}$, we have $-(1-$ p) $\gamma \int_{a}^{b} S^{*}(x) \phi_{i}(x) \mathrm{d} x<0$. Consequently, if $\frac{2-c_{i}}{1-\beta}<z_{i}^{L A} \leq b$ and $\frac{1-c_{i}}{1-\beta}<\frac{a+b}{2}$, we have $\frac{\partial z_{i}^{L A}}{\partial l}<0$.

Proposition 4 indicates that an increase in the the risk coefficient $l$ for losses raises the reservation value of low-value product and reduces the reservation value of highvalue product. This means that when the coefficient of risk attitude for losses is very high, the consumer would raise the purchase threshold for low-value product and reduce the purchase threshold for high-value product.

Finally, we can investigate the effect of coefficient of loss aversion on the reservation value $z_{i}^{L A}$.

Proposition 5. Holding other parameters of the model constant, the reservation value of the firm's product under the prospect utility is decreasing with respect to the coefficient of loss aversion.

Proof. By taking the partial derivative of the variable $z_{i}^{L A}$ of equation $D^{p}\left(z_{i}^{L A}\right)=0$ with respect to $\gamma$, we obtain:

$$
\begin{aligned}
& -\frac{\partial z_{i}^{L A}}{\partial \gamma}-\beta p z \phi_{i}(z) \frac{\partial z_{i}^{L A}}{\partial \gamma}-p g \int_{\frac{z+c_{i}}{\beta}}^{b}\left(\beta x-c_{i}-z\right)^{g-1} \phi_{i}(x) \frac{\partial z_{i}^{L A}}{\partial \gamma} \mathrm{d} x \\
& -p \int_{z}^{\frac{z+c_{i}}{\beta}}\left(z-\beta x+c_{i}\right)^{l} \phi_{i}(x) \mathrm{d} x-p \gamma l \int_{z}^{\frac{z+c_{i}}{\beta}}\left(z-\beta x+c_{i}\right)^{l-1} \phi_{i}(x) \frac{\partial z_{i}^{L A}}{\partial \gamma} \mathrm{d} x \\
& -p \int_{a}^{z}\left(z-\beta z+c_{i}\right)^{l} \phi_{i}(x) \mathrm{d} x-p \gamma l(1-\beta) \int_{a}^{z}\left(z-\beta z+c_{i}\right)^{l-1} \phi_{i}(x) \frac{\partial z_{i}^{L A}}{\partial \gamma} \mathrm{d} x \\
& -(1-p) \int_{a}^{b}\left(x-\beta x+c_{i}\right)^{l} \phi_{i}(x) \mathrm{d} x=0 .
\end{aligned}
$$

Moreover, we have:

$$
\frac{\partial z_{i}^{L A}}{\partial \gamma}=\frac{-p \int_{z}^{\frac{z+c_{i}}{\beta}} W_{1} \phi_{i}(x) \mathrm{d} x-p \int_{a}^{z} W_{2} \phi_{i}(x) \mathrm{d} x-(1-p) \int_{a}^{b} W_{3} \phi_{i}(x) \mathrm{d} x}{N}<0,
$$

where:

$$
\begin{aligned}
N= & 1+\beta p z \phi_{i}(z)+p g \int_{\frac{z+c_{i}}{\beta}}^{b}\left(\beta x-c_{i}-z\right)^{g-1} \phi_{i}(x) \mathrm{d} x \\
& +p \gamma l \int_{z}^{\frac{z+c_{i}}{\beta}}\left(z-\beta x+c_{i}\right)^{l-1} \phi_{i}(x) \mathrm{d} x+p \gamma l(1-\beta) \int_{a}^{z}\left(z-\beta z+c_{i}\right)^{l-1} \phi_{i}(x) \mathrm{d} x
\end{aligned}
$$


and $W_{1}=\left(z-\beta x+c_{i}\right)^{l}, W_{2}=\left(z-\beta z+c_{i}\right)^{l}, W_{3}=\left(x-\beta x+c_{i}\right)^{l}$.

From Proposition 5, it shows that the reservation value of the firm's product is decreasing with the coefficient of loss aversion, that is to say, the consumer who has more sensitive to the loss will search for much less products. Hence, the above propositions make it clear that risk attitude for searching high-value product and loss aversion would indeed lead to 'too little search.'

\section{Numerical examples}

In this section, some numerical examples are given to illustrate the reservation values under the models without and with prospect utility and examine the effects of risk attitude for gains, risk attitude for losses, and loss aversion on the optimal search strategy.

Suppose a consumer must decide to buy one among three products. Because the three products are differentiated but substitutable, the consumer only knows different uncertainty distributions about the three products. Without loss of generality, suppose the consumer's valuations for the three products are linear uncertain variables $\mathcal{L}(1,9)$, $\mathcal{Z}(1,4,9)$, and $\mathcal{Z}(1,7,9)$, respectively. Let $\beta=0.9, p=0.7, c_{1}=0.5, c_{2}=1$, and $c_{3}=1.5$. By Equation 9, the reservation values of products can be calculated as $z_{1}^{L N}=3.5$, $z_{2}^{L N}=2.4$, and $z_{3}^{L N}=3.6$. Therefore, the consumer can rank the products according to their respective reservation value so that $z_{3}^{L N}>z_{1}^{L N}>z_{2}^{L N}$. Then, the consumer can evaluate three products in accordance with the order of the reservation value and make a purchase decision through the optimal search strategy without prospect utility. Similarly, we can also obtain the prospect utility case by the equation $D^{p}\left(z_{i}^{L A}\right)=0$.

To illustrate the reservation values under the models without and with prospect utility numerically, suppose the parameters $g=0.5, l=0.5$, and $\gamma=2$. Without loss of generality, we only consider the first product when the parameters $\beta=0.9, p=0.7$, and $c_{1}=0.5$ remain unchanged. From Figure 1 , we observe that $z_{1}^{L A}<z_{1}^{L N}$. However, the sufficient condition $7.6<z_{1}^{L N}<9$ of Proposition 2 cannot hold. It implies that the reservation value $z_{i}^{L N}$ which does not satisfy the sufficient condition may give rise to the result of Proposition 2.

We find our key results continue to hold in our numerical studies. When the risk coefficient $g$ for gains can be chosen in the set $\{0.5,0.7,0.9\}$, Figure 2 reports our findings for $\beta=0.9, p=0.7, c_{1}=0.5, l=0.5$, and $\gamma=2$, which is representative, and other parameter combinations considered in our studies also yield similar results. As expected, Figure 2 shows the reservation value of low-value product which satisfies $1 \leq z_{1}^{L A}<5.6$ will be increased with the coefficient of risk attitude for gains. When the risk coefficient $l$ for losses can be chosen in the set $\{0.5,0.7,0.9\}$ and the other parameters $\beta=0.9, p=0.7$, $c_{1}=0.5, g=0.5$, and $\gamma=2$ remain unchanged, Figure 3 shows that the reservation value of low-value product which satisfies $1 \leq z_{1}^{L A}<5$ is increasing with respect to the coefficient of risk attitude for losses.

Finally, the effect of loss aversion on the reservation value can be examined. Without loss of generality, suppose the coefficient $\gamma$ of loss aversion can be chosen in the set $\{1.5,2,2.5\}$ and the other parameters $\beta=0.9, p=0.7, c_{1}=0.5, g=0.5$, and $l=0.5$ remain unchanged. From Figure 4 , it is apparent that the reservation value of the firm's product is decreasing with the coefficient of loss aversion. 


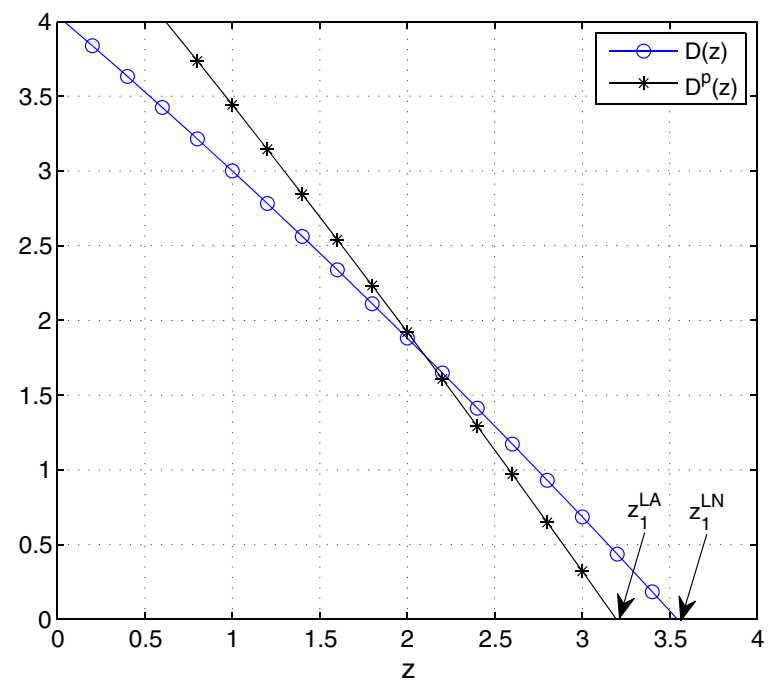

Figure 1 A comparison between reservation values.

\section{Conclusion}

This paper presented a consumer search model with prospect utility, in which the subjective valuations of the consumer to every firm's product were assumed to be independent but not identically distributed uncertain variables, and the valuation of a reserved product with probabilistic stockout was consequently characterized as an uncertain random variable. An optimal stopping rule and an optimal search strategy were proposed to maximize the benefit of the consumer. Moreover, we studied the consumer's purchase threshold in the model without and with prospect utility. The results demonstrated that the prospect utility will lead to the low purchase threshold. We found that the consumer's purchase threshold is decreasing with search cost and is increasing with the probability of stockout. Furthermore, we also found that the product that has a good reputation will become the preferred product in the search process. In addition, the risk

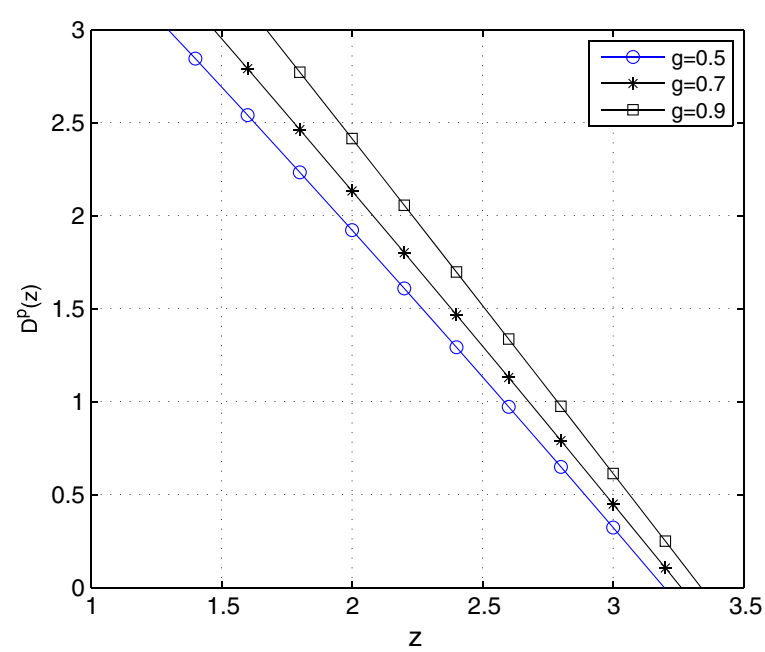

Figure 2 Effect of risk attitude for gains on the reservation value of low-value product. 


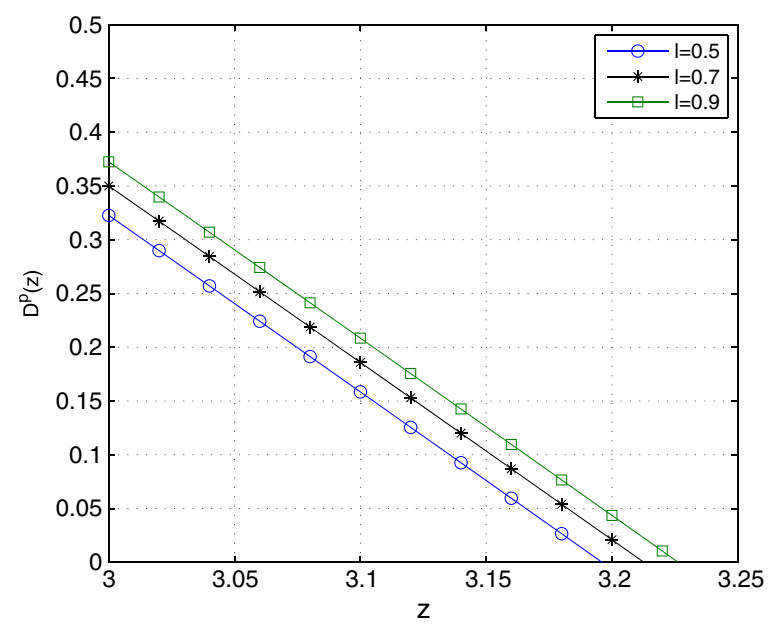

Figure 3 Effect of risk attitude for losses on the reservation value of low-value product.

coefficients for gains and losses will raise the consumer's purchase threshold for lowvalue product, while they will reduce the purchase threshold for high-value product. To provide a simple explanation for why consumers generally stop searching too early, we showed that the loss aversion will reduce the consumer's purchase threshold. Finally, some numerical examples were given to illustrate the effectiveness of the proposed models and conclusions.

In the future work, we can incorporate advertisement into consumer search in the presence of loss aversion. For instance, firms will post-advertise to improve their goodwill and induce consumers to buy their products. In addition, it is also interesting to study consumer inertia in the dynamic search and examine the effects of prospect utility on the consumer inertia.

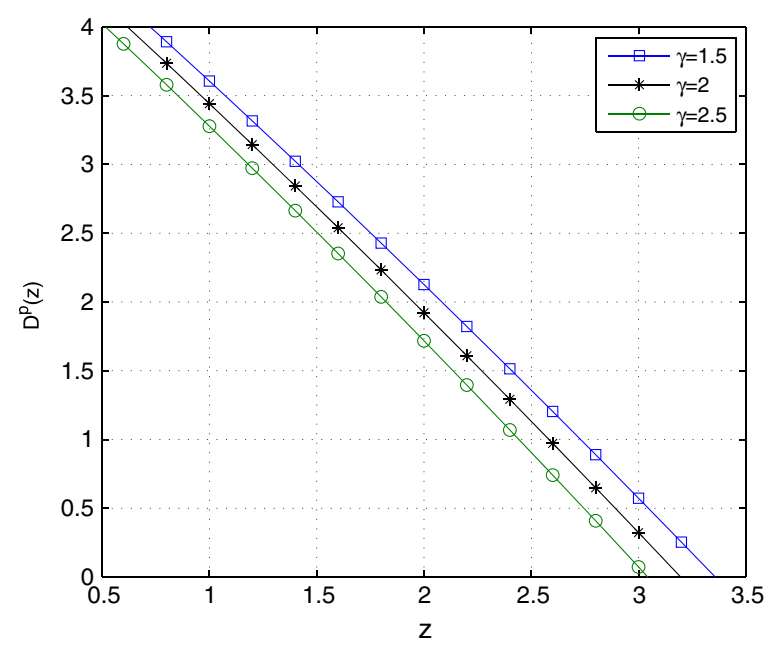

Figure 4 Effect of loss aversion on the reservation value. 


\section{Endnotes}

${ }^{a}$ This statistical result is publicly available at http://hbr.org/2004/05/stock-outs-causewalkouts.

b This means the product which obtained low score. Similarly, 'high-value product' means the product which obtained high score.

\section{Competing interests}

The authors declare that they have no competing interests.

\section{Acknowledgements}

This work is supported by the National Natural Science Foundation of China (Nos. 71071106 and 71371133) and supported partially by the Specialized Research Fund for the Doctoral Program of Higher Education of China (No. 20120032110071) and the Program for New Century Excellent Talents in Universities of China.

Received: 12 December 2014 Accepted: 6 February 2015

Published online: 12 March 2015

\section{References}

1. Schunk, D: Behavioral heterogeneity in dynamic search situations: theory and experimental evidence. J. Econ. Dyn. Control. 33(9), 1719-1738 (2009)

2. Tversky, A: Prospect theory: an analysis of decision under risk. Econometrica: J. Econometric Soc. 47(2), 263-291 (1979)

3. Tversky, A, Kahneman, D: Loss aversion in riskless choice: a reference-dependent model. Q. J. Econ. 106(4), 1039-1061 (1991)

4. Tversky, A, Kahneman, D: Advances in prospect theory: cumulative representation of uncertainty. J. Risk Uncertainty. 5(4), 297-323 (1992)

5. Liu, B: Uncertainty Theory. 2nd edn. Springer, Berlin (2007)

6. Liu, Y: Uncertain random variables: a mixture of uncertainty and randomness. Soft Comput. 17(4), 625-634 (2013)

7. Branco, F, Sun, M, Villas-Boas, JM: Optimal search for product information. Manage. Sci. 58(11), 2037-2056 (2012)

8. Schunk, D, Winter, J: The relationship between risk attitudes and heuristics in search tasks: a laboratory experiment. J. Econ. Behavior Organization. 71(2), 347-360 (2009)

9. Stahl, DO: Oligopolistic pricing with sequential consumer search. Am. Econ. Rev. 79(4), 700-712 (1989)

10. Cachon, GP, Terwiesch, $C, X u, Y$ : On the effects of consumer search and firm entry in a multiproduct competitive market. Marketing Sci. 27(3), 461-473 (2008)

11. Arbatskaya, M: Ordered search. RAND J. Econ. 38(1), 119-126 (2007)

12. Wilson, CM: Ordered search and equilibrium obfuscation. Int. J. Ind. Organization. 28(5), 496-506 (2010)

13. Zhou, J: Ordered search in differentiated markets. Int. J. Ind. Organization. 29(2), 253-262 (2011)

14. Chen, Y, Zhang, T: Equilibrium price dispersion with heterogeneous searchers. Int. J. Ind. Organization. 29(6), 645-654 (2011)

15. Haan, MA, Moraga-González, JL: Advertising for attention in a consumer search model. Econ. J. 121(552), 552-579 (2011)

16. Chen, Y, He, C: Paid placement: advertising and search on the internet. Econ. J. 121(556), 309-328 (2011)

17. Hardie, BG, Johnson, EJ, Fader, PS: Modeling loss aversion and reference dependence effects on brand choice. Marketing Sci. 12(4), 378-394 (1993)

18. Weng, Z: Consumer search and firm-worker reciprocity: a behavioral approach. PhD thesis, The Ohio State University (2010)

19. Zhou, J: Reference dependence and market competition. J. Econ. Manage. Strategy. 20(4), 1073-1097 (2011)

20. Kuksov, D, Wang, K: The bright side of loss aversion in dynamic and competitive markets. Marketing Sci. 33(5), 693-711 (2014)

21. Bowman, D, Minehart, D, Rabin, M: Loss aversion in a consumption-savings model. J. Econ. Behavior Organization. 38(2), 155-178 (1999)

22. Popescu, I, Wu, Y: Dynamic pricing strategies with reference effects. Operations Res. 55(3), 413-429 (2007)

23. Foellmi, R, Rosenblatt-Wisch, R, Schenk-Hoppé, KR: Consumption paths under prospect utility in an optimal growth model. J. Econ. Dyn. Control. 35(3), 273-281 (2011)

24. $\mathrm{Mu}, \mathrm{R}$, Lan, Y, Tang, W: An uncertain contract model for rural migrant worker's employment problems. Fuzzy Optimization Decision Making. 12(1), 29-39 (2013)

25. Lan, Y, Du, H: A warranty contract model with uncertain demands. Inf.-An Int. Interdisplinary J. 16(2), 1037-1045 (2013)

26. Zhou, C, Tang, W, Zhao, R: An uncertain search model for recruitment problem with enterprise performance. J. Intell. Manuf. (2014). doi:10.1007/s10845-014-0997-1

27. Wang, $X$, Lan, $Y$, Wang, J: An uncertain wage contract model with adverse selection and moral hazard. J. Appl. Mathematics. 2014(Article ID 282867), 1-9 (2014)

28. Wu, X, Zhao, R, Tang, W: Optimal contracts for the agency problem with multiple uncertain information. Knowledge-Based Syst. 59, 161-172 (2014)

29. Liu, Y: Uncertain random programming with applications. Fuzzy Optimization Decision Making. 12(2), $153-169$ (2013)

30. Gao, J, Yao, K: Some concepts and theorems of uncertain random process. Int. J. Intell. Syst. to be published (2014)

31. Liu, B: Uncertain random graph and uncertain random network. J. Uncertain Syst. 8(1), 3-12 (2014)

32. Sheng, Y, Yao, K: Some formulas of variance of uncertain random variable. J. Uncertainty Anal. Appl. 2(1), 12 (2014)

33. Zhou, J, Yang, F, Wang, K: Multi-objective optimization in uncertain random environments. Fuzzy Optimization Decision Making. 13(4), 397-413 (2014) 
34. Liu, B: Some research problems in uncertainty theory. J. Uncertain Syst. 3(1), 3-10 (2009)

35. Peng, Z, Iwamura, K: A sufficient and necessary condition of uncertainty distribution. J. Interdisciplinary Mathematics. 13(3), 277-285 (2010)

36. Liu, B: Uncertainty Theory: A Branch of Mathematics for Modeling Human Uncertainty. Springer, Berlin (2010)

37. Liu, B: Toward uncertain finance theory. J. Uncertainty Anal. Appl. 1, 1 (2013)

38. Liu, Y, Ha, M: Expected value of function of uncertain variables. J. Uncertain Syst. 4(3), 181-186 (2010)

39. Gao, Z, Guo, H: Some properties of uncertain dominance. Int. Inf. Inst. (Tokyo). Inf. 15(12), 168-173 (2013)

40. Hou, Y: Subadditivity of chance measure. Journal of Uncertainty Analysis and Applications. 2, 14 (2014)

41. DeGroot, MH: Optimal Statistical Decisions. (John Wiley \& Sons, Inc., Hoboken (2004)

42. Lippman, SA, McCall, JJ: The economics of job search: a survey. Econ. Inquiry. 14(2), 155-189 (1976)

43. Weitzman, ML: Optimal search for the best alternative. Econometrica: J. Econometric Soc. 47(3), 641-654 (1979)

44. Abdellaoui, M, Bleichrodt, $\mathrm{H}$, Paraschiv, C: Loss aversion under prospect theory: a parameter-free measurement. Manage. Sci. 53(10), 1659-1674 (2007)

Submit your manuscript to a SpringerOpen ${ }^{\odot}$ journal and benefit from:

- Convenient online submission

- Rigorous peer review

- Immediate publication on acceptance

- Open access: articles freely available online

- High visibility within the field

- Retaining the copyright to your article

Submit your next manuscript at $\gg$ springeropen.com 